Bull. Austral. Math. Soc.

$47 \mathrm{H} 99,47 \mathrm{~J} 20,49 \mathrm{~s} 40$

VoL. 73 (2006) [307-317]

\title{
THE GENERALISED $f$-PROJECTION OPERATOR WITH AN APPLICATION
}

\author{
Ke-QING Wu and Nan-Jing Huang
}

\begin{abstract}
In this paper, we introduce a new concept of generalised $f$-projection operator which extends the generalised projection operator $\pi_{K}: B^{*} \rightarrow K$, where $B$ is a reflexive Banach space with dual space $B^{*}$ and $K$ is a nonempty, closed and convex subset of $B$. Some properties of the generalised $f$-projection operator are given. As an application, we study the existence of solution for a class of variational inequalities in Banach spaces.
\end{abstract}

\section{INTRODUCTION AND PRELIMINARIES}

Let $B$ be a Banach space with dual space $B^{*}$. As usual, $\langle\varphi, x\rangle$, denotes the duality pairing of $B^{*}$ and $B$, where $\varphi \in B^{*}$ and $x \in B$. If $B$ is a Hilbert space, $\langle\varphi, x\rangle$ denotes an inner product on $B$. Let $K$ be a nonempty, closed and convex subset of $B$. The metric projection operator $P_{K}: B^{*} \rightarrow K$ has been used in many areas such as optimisation theory, fixed point theory, nonlinear programming, game theory, variational inequality, and complementarity problems, et cetera (see, for example, $[10,11,12,13,14,16,17$, 20] and the references therein).

In 1994, Alber [1] introduced the generalised projections $\pi_{K}: B^{*} \rightarrow K$ and $\Pi_{K}$ : $B \rightarrow K$ from Hilbert spaces to uniformly convex and uniformly smooth Banach spaces and studied their properties in detail. In [2], Alber presented some applications of the generalised projections to approximate solving variational inequalities and Von-Neumann intersection problem in Banach spaces. Recently, Li [17] extended the definition of the generalised projection operator $\pi_{K}: B^{*} \rightarrow K$, where $B$ is a reflexive Banach space with dual space $B^{*}$ and $K$ is a nonempty, closed and convex subset of $B$ and studied some properties of the generalised projection operator with applications to solving the variational inequality in Banach spaces. Some related works, we refer to $[3,4,5,6,7$, $8,9]$ and the references therein.

Received 9th January, 2006

This work was supported by the National Natural Science Foundation of China, the Applied Research Project of Sichuan Province (05JY029-009-1) and the Educational Science Foundation of Chongqing (KJ051307).

Copyright Clearance Centre, Inc. Serial-fee code: 0004-9727/06 \$A2.00+0.00. 
Motivated and inspired by the above works, in this paper, we introduce and study a new class of generalised $f$-projection operator in Banach spaces, which extends the definition of the generalised projection operators introduced by Alber [1] and $\mathrm{Li}[17]$. Some properties of the generalised $f$-projection operator are given. As an application, we study the existence of solution to a class of variational inequalities.

Let $B$ be a Banach space with dual space $B^{*}$. The normalised duality mapping $J: B \rightarrow 2^{B^{*}}$ is defined by

$$
J(x)=\left\{j(x) \in B^{*}:\langle j(x), x\rangle=\|j(x)\| \cdot\|x\|=\|x\|^{2}=\|j(x)\|^{2}\right\},
$$

where $(\cdot, \cdot\rangle$ denotes the duality pairing of $B^{*}$ and $B$. Without confusion, one understands that $\|j(x)\|$ is the $B^{*}$-norm and $\|x\|$ is the $B$-norm. Many properties of the normalised duality mapping $J$ can be found in Takahashi [18] or Vainberg [19]. We list some properties below for easy reference:

(i) $J$ is a monotone and bounded operator in arbitrary Banach spaces;

(ii) $J$ is a strictly monotone operator in strictly convex Banach spaces;

(iii) $J$ is a continuous operator in smooth Banach spaces;

(iv) $J$ is a uniformly continuous operator on each bounded set in uniformly smooth Banach spaces;

(v) $J$ is the identity operator in Hilbert spaces, that is, $J=I_{H}$;

(vi) $J(x)=\partial\left(\|x\|^{2} / 2\right)$, where $\partial\left(\|x\|^{2} / 2\right)$ denotes subdifferential of $\|x\|^{2} / 2$ at $x$.

Let $G: B^{*} \times B \rightarrow R \cup\{+\infty\}$ be a functional defined as follows:

$$
G(\varphi, x)=\|\varphi\|^{2}-2\langle\varphi, x\rangle+\|x\|^{2}+f(x)
$$

where $\varphi \in B^{*}, x \in B$ and $f: B \rightarrow R \cup\{+\infty\}$ is proper, convex, lower semi-continuous, and bounded from below. It is easy to see that

$$
G(\varphi, x) \geqslant(\|\varphi\|-\|x\|)^{2}+f(x) .
$$

From the definitions of $G$ and $f$, it is easy to see that the $G$ has the following properties:

(vii) $G(\varphi, x)$ is convex and continuous with respect to $\varphi$ when $x$ is fixed;

(viii) $G(\varphi, x)$ is convex and lower-semi-continuous with respect to $x$ when $\varphi$ is fixed;

(ix) $\quad(\|\varphi\|-\|x\|)^{2}+f(x) \leqslant G(\varphi, x) \leqslant(\|\varphi\|+\|x\|)^{2}+f(x)$.

LEMMA 1.1. ([15, p. 94].) Let $X$ be a Banach space. The following conditions are equivalent.

(1) $X$ is strictly convex; 
(2) If $x, y \in X$ and $\|x+y\|=\|x\|+\|y\|$, then $x=0$ or $y=0$ or $y=\alpha x$ for some $\alpha>0$.

Definition 1.1: Let $\mathrm{B}$ be a Banach space with dual space $B^{*}$. Let $K$ be a nonempty, closed and convex subset of B. We say that $\pi_{K}^{f}: B^{*} \rightarrow 2^{K}$ is a generalised $f$-projection operator if

$$
\pi_{K}^{f} \varphi=\left\{u \in K: G(\varphi, u)=\inf _{y \in K} G(\varphi, y)\right\} \quad \forall \varphi \in B^{*} .
$$

REMARK 1.1. If $f(x)=0$ for all $x \in B$, then the generalised $f$-projection operator reduces to the generalised projection operator defined by Alber [1] and Li [17].

\section{Properties OF THE GENERALISEd $f$-PROJECTION $\pi_{K}^{f}$}

The following theorem shows that the operator $\pi_{K}^{f}$ is well defined for reflexive Banach spaces.

Theorem 2.1. If $B$ is a reflexive Banach space with dual space $B^{*}$ and $K$ is a nonempty closed convex subset of $B$, then $\pi_{K}^{f} \varphi \neq \emptyset$ for all $\varphi \in B^{*}$.

Proof: For any given $\varphi \in B^{*}$ and $x \in B$, we have

$$
(\|\varphi\|-\|x\|)^{2}+f(x) \leqslant G(\varphi, x) \leqslant(\|\varphi\|+\|x\|)^{2}+f(x) .
$$

Since $f$ is bounded from below, it follows that, for any given $\varphi \in B^{*}, \inf _{y \in K} G(\varphi, y)$ is finite and so there exist a sequence $\left\{x_{n}\right\} \in K$ such that

$$
\lim _{n \rightarrow \infty} G\left(\varphi, x_{n}\right)=\inf _{y \in K} G(\varphi, y) .
$$

Let $\inf _{y \in K} G(\varphi, y)=a$. Then for any given $\varepsilon>0$, there exists $N>0$ such that

$$
\left|G\left(\varphi, x_{n}\right)-a\right|<\varepsilon
$$

for all $n \geqslant N$. Thus,

$$
\left(\|\varphi\|-\left\|x_{n}\right\|\right)^{2}+f\left(x_{n}\right)-a<\varepsilon .
$$

Since $f(x)$ is bounded from below, there exists $L \in R$ such that

$$
\left(\|\varphi\|-\left\|x_{n}\right\|\right)^{2}+L-a<\varepsilon .
$$

On the other hand,

$$
\begin{aligned}
a=\inf _{y \in K} G(\varphi, y) & =\inf _{y \in K}\left\{\|\varphi\|^{2}-2\left\langle\varphi, x_{n}\right\rangle+\left\|x_{n}\right\|^{2}+f\left(x_{n}\right)\right\} \\
& \geqslant \inf _{y \in K}\left\{\|\varphi\|^{2}-2\left\langle\varphi, x_{n}\right\rangle+\left\|x_{n}\right\|^{2}\right\}+L \\
& \geqslant \inf _{y \in K}\left(\|\varphi\|-\left\|x_{n}\right\|\right)^{2}+L \\
& \geqslant L .
\end{aligned}
$$


From (2.2) and (2.3), we know that $\left\{x_{n}\right\}$ is bounded. Since $B$ is reflexive, there exist a subsequence of $\left\{x_{n}\right\}$, which after relabelling we again denote by $\left\{x_{n}\right\}$, and a point $x_{0} \in K$ such that $\left\{x_{n}\right\}$ converges weakly to $x_{0}$. For each given $\varphi$, since $G(\varphi, x)$ is convex and lower-semi-continuous with respect to $x$, we know that $G(\varphi, x)$ is weakly lower-semicontinuous with respect to $x$. Thus, we have

$$
G\left(\varphi, x_{0}\right) \leqslant \liminf _{n \rightarrow \infty} G\left(\varphi, x_{n}\right)=\lim _{n \rightarrow \infty} G\left(\varphi, x_{n}\right)=\inf _{y \in K} G(\varphi, y)
$$

and so $x_{0} \in \pi_{K}^{f} \varphi$. Therefore, $\pi_{K}^{f} \varphi \neq \emptyset$. This completes the proof.

THEOREM 2.2. If $B$ is a refexive Banach space with dual space $B^{*}$ and $K$ is a nonempty, closed and convex subset of $B$, then the following properties hold:

$\left(f_{1}\right)$ For any given $\varphi \in B^{*}, \pi_{K}^{f} \varphi$ is a nonempty, closed, bounded, and convex subset of $K$;

$\left(f_{2}\right) \pi_{K}^{f}$ is monotone, that is, for any $\varphi_{1}, \varphi_{2} \in B^{*}, x_{1} \in \pi_{K}^{f} \varphi_{1}$ and $x_{2} \in \pi_{K}^{f} \varphi_{2}$,

$$
\left\langle x_{1}-x_{2}, \varphi_{1}-\varphi_{2}\right\rangle \geqslant 0
$$

$\left(f_{3}\right)$ If $B$ is smooth, then for any given $\varphi \in B^{*}, x \in \pi_{K}^{f} \varphi$ if and only if

$$
2\langle\varphi-J(x), x-y\rangle+f(y)-f(x) \geqslant 0
$$

for all $y \in K$;

$\left(f_{4}\right)$ If $K$ is a closed convex cone and $f: K \rightarrow R \cup\{+\infty\}$ is positively homogeneous, that is, $f(t x)=t f(x)$ for all $t>0$ and $x \in K$, then for any $\varphi \in B^{*}$ and $x_{1}, x_{2} \in \pi_{K}^{f} \varphi$, we have $x_{1} \neq \mu x_{2}$ for all $\mu \in(0,+\infty)$ with $\mu \neq 1$;

$\left(f_{5}\right)$ If $K$ is a closed convex cone, $f: K \rightarrow R \cup\{+\infty\}$ is positively homogeneous and $B$ is strictly convex, then the operator $\pi_{K}^{f}: B^{*} \rightarrow K$ is single-valued.

Proof: $\left(f_{1}\right)$ For any point $\varphi \in B^{*}$, Theorem 2.1 implies that $\pi_{K}^{f} \varphi$ is nonempty. Since $f$ is bounded from below and $G(\varphi, x) \geqslant(\|\varphi\|-\|x\|)^{2}+f(x)$, it is easy to see that $\pi_{K}^{f} \varphi$ is bounded. Next we prove that $\pi_{K}^{f} \varphi$ is closed. Suppose $\left\{x_{n}\right\} \in \pi_{K}^{f} \varphi$ and $x_{n} \rightarrow x_{0}$ as $n \rightarrow \infty$. By property (viii) of the functional $G$, we have

$$
G\left(\varphi, x_{0}\right) \leqslant \liminf _{n \rightarrow \infty} G\left(\varphi, x_{n}\right)=\lim _{n \rightarrow \infty} G\left(\varphi, x_{n}\right)=\inf _{y \in K} G(\varphi, y) .
$$

Thus, $x_{0} \in \pi_{K}^{f} \varphi$ and so $\pi_{K}^{f} \varphi$ is closed. Finally, we prove that $\pi_{K}^{f} \varphi$ is convex. Suppose $x_{1}, x_{2} \in \pi_{K}^{f} \varphi$ and $0 \leqslant t \leqslant 1$. From property (viii) of the functional $G$, we have

$$
\begin{aligned}
G\left(\varphi, t x_{1}+(1-t) x_{2}\right) & \leqslant t G\left(\varphi, x_{1}\right)+(1-t) G\left(\varphi, x_{2}\right) \\
& =t \inf _{y \in K} G(\varphi, y)+(1-t) \inf _{y \in K} G(\varphi, y) \\
& =\inf _{y \in K} G(\varphi, y)
\end{aligned}
$$


and so $t x_{1}+(1-t) x_{2} \in \pi_{K}^{f} \varphi$. This implies that $\pi_{K}^{f} \varphi$ is convex.

$\left(f_{2}\right)$ For any $\varphi_{1}, \varphi_{2} \in B^{*}, x_{1} \in \pi_{K}^{f} \varphi_{1}$ and $x_{2} \in \pi_{K}^{f} \varphi_{2}$, from definition $\pi_{K}^{f}$, we have

$$
\left\|\varphi_{1}\right\|^{2}-2\left\langle\varphi_{1}, x_{1}\right\rangle+\left\|x_{1}\right\|^{2}+f\left(x_{1}\right) \leqslant\left\|\varphi_{1}\right\|^{2}-2\left\langle\varphi_{1}, x_{2}\right\rangle+\left\|x_{2}\right\|^{2}+f\left(x_{2}\right)
$$

and

$$
\left\|\varphi_{2}\right\|^{2}-2\left\langle\varphi_{2}, x_{2}\right\rangle+\left\|x_{2}\right\|^{2}+f\left(x_{2}\right) \leqslant\left\|\varphi_{2}\right\|^{2}-2\left\langle\varphi_{2}, x_{1}\right\rangle+\left\|x_{1}\right\|^{2}+f\left(x_{1}\right) .
$$

It follows from (2.4) and (2.5) that $\pi_{K}^{f}$ is monotone.

$\left(f_{3}\right)$ We first prove that $x \in \pi_{K}^{f} \varphi$ implies that

$$
2\langle\varphi-J(x), x-y\rangle+f(y)-f(x) \geqslant 0, \quad \forall y \in K .
$$

In fact, for any $y \in K$ and $t \in(0,1]$, it follows from the definition of $\pi_{K}^{f} \varphi$ that

$$
G(\varphi, x) \leqslant G(\varphi, x+t(y-x)) .
$$

Thus,

$$
\begin{aligned}
\|\varphi\|^{2}-2\langle\varphi, x\rangle+\|x\|^{2}+f(x) \leqslant\|\varphi\|^{2}-2\langle\varphi, x+ & t(y-x)\rangle \\
& +\|x+t(y-x)\|^{2}+f(x+t(y-x))
\end{aligned}
$$

and so

$$
\begin{aligned}
2\langle\varphi, t(y-x)\rangle+\|x\|^{2}+f(x) & \leqslant\|x+t(y-x)\|^{2}+f(x+t(y-x)) \\
& \leqslant\|x+t(y-x)\|^{2}+(1-t) f(x)+t f(y) .
\end{aligned}
$$

It follows that

$$
2\langle\varphi, t(y-x)\rangle+\|x\|^{2} \leqslant\|x+t(y-x)\|^{2}+t(f(y)-f(x)) .
$$

Now from the properties of the normalised duality mapping, we have

$$
\langle J(x+t(y-x)),-t(y-x)\rangle \leqslant \frac{1}{2}\|x\|^{2}-\frac{1}{2}\|x+t(y-x)\|^{2} .
$$

By (2.6), we get

$$
2\langle J(x+t(y-x)), y-x\rangle \geqslant f(x)-f(y)+2\langle(\varphi, y-x)\rangle .
$$

Since $B$ is smooth, we know that $J$ is demi-continuous. Letting $t \rightarrow 0$ in the above inequality, we have

$$
2\langle J(x)-\varphi, y-x\rangle+f(y)-f(x) \geqslant 0 .
$$


Conversely, suppose

$$
2\langle J(x)-\varphi, y-x\rangle+f(y)-f(x) \geqslant 0 \text { for all } y \in K
$$

Then

$$
\|y\|^{2}-\|x\|^{2} \geqslant 2\langle J(x), y-x\rangle \geqslant 2\langle\varphi, y-x\rangle+f(x)-f(y)
$$

which implies that $G(\varphi, x) \leqslant G(\varphi, y)$ for all $y \in K$, that is, $x \in \pi_{K}^{f} \varphi$.

$\left(f_{4}\right)$ Assume $x_{1}, x_{2} \in \pi_{K}^{f} \varphi$ and $x_{1}=\mu x_{2}$ for some real number $\mu>0$ with $\mu \neq 1$. Then $G\left(\varphi, x_{1}\right)=G\left(\varphi, x_{2}\right)$ and so

$$
2\left\langle\varphi, x_{2}-x_{1}\right\rangle=\left\|x_{2}\right\|^{2}+f\left(x_{2}\right)-\left\|x_{1}\right\|^{2}-f\left(x_{1}\right) .
$$

Replacing $x_{1}$ by $\mu x_{2}$ in above equality, we have

$$
2(1-\mu)\left\langle\varphi, x_{2}\right\rangle=\left(1-\mu^{2}\right)\left\|x_{2}\right\|^{2}+(1-\mu) f\left(x_{2}\right) .
$$

Since $\mu \neq 1$, we obtain

$$
2\left\langle\varphi, x_{2}\right\rangle=(1+\mu)\left\|x_{2}\right\|^{2}+f\left(x_{2}\right)
$$

Let

$$
x_{3}=\left(x_{2}+x_{1}\right) / 2=((1+\mu) / 2) x_{2} .
$$

It follows from $\left(f_{1}\right)$ that $x_{3} \in \pi_{K}^{f} \varphi$ and so $G\left(\varphi, x_{2}\right)=G\left(\varphi, x_{3}\right)$. Similarly, we can get

$$
2\left\langle\varphi, x_{2}\right\rangle=\left(1+\frac{1}{2}(1+\mu)\right)\left\|x_{2}\right\|^{2}+f\left(x_{2}\right) \text {. }
$$

Now (2.7) and (2.8) imply that $1+\mu=1+(1+\mu) / 2$ and so $\mu=1$, which is a contradiction to $\mu \neq 1$. Thus, $\left(f_{4}\right)$ is true.

$\left(f_{5}\right)$ Suppose there exists $\varphi \in B^{*}$ such that $\pi_{K} \varphi$ is not a singleton. Then for any $x_{1}, x_{2} \in \pi_{K}^{f} \varphi$ and $x_{1} \neq x_{2}$, we have $G\left(\varphi, x_{1}\right)=G\left(\varphi, x_{2}\right)$. This implies

$$
2\left\langle\varphi, x_{2}-x_{1}\right\rangle=\left\|x_{2}\right\|^{2}+f\left(x_{2}\right)-\left\|x_{1}\right\|^{2}-f\left(x_{1}\right)
$$

By property $\left(f_{1}\right)$, for any $t \in[0,1]$, we know that $x_{1}+t\left(x_{2}-x_{1}\right) \in \pi_{K} \varphi$. Since $G\left(\varphi, x_{1}\right.$ $\left.+t\left(x_{2}-x_{1}\right)\right)=G\left(\varphi, x_{1}\right)$,

(2.10) $2 t\left(\varphi, x_{2}-x_{1}\right\rangle=\left\|x_{1}+t\left(x_{2}-x_{1}\right)\right\|^{2}+f\left(x_{1}+t\left(x_{2}-x_{1}\right)\right)-\left\|x_{1}\right\|^{2}-f\left(x_{1}\right)$.

Combining (2.9) and (2.10), we have

$$
\begin{aligned}
t\left(\left\|x_{2}\right\|^{2}+\right. & \left.f\left(x_{2}\right)-\left\|x_{1}\right\|^{2}-f\left(x_{1}\right)\right) \\
& =\left\|x_{1}+t\left(x_{2}-x_{1}\right)\right\|^{2}+f\left(x_{1}+t\left(x_{2}-x_{1}\right)\right)-\left\|x_{1}\right\|^{2}-f\left(x_{1}\right) \\
& \leqslant\left\|x_{1}+t\left(x_{2}-x_{1}\right)\right\|^{2}+t\left(\left(x_{2}-f\left(x_{1}\right)\right)\right)-\left\|x_{1}\right\|^{2} .
\end{aligned}
$$


This implies that

$$
t\left\|x_{2}\right\|^{2}+(1-t)\left\|x_{1}\right\|^{2} \leqslant\left\|x_{1}+t\left(x_{2}-x_{1}\right)\right\|^{2}
$$

and so

$$
\begin{aligned}
\left\|x_{1}+t\left(x_{2}-x_{1}\right)\right\|^{2} & \leqslant\left(t\left\|x_{2}\right\|+(1-t)\left\|x_{1}\right\|\right)^{2} \\
& =t^{2}\left\|x_{2}\right\|^{2}+2 t(1-t)\left\|x_{1}\right\|\left\|x_{2}\right\|+(1-t)^{2}\left\|x_{1}\right\|^{2} \\
& \leqslant t\left\|x_{2}\right\|^{2}+(1-t)\left\|x_{1}\right\|^{2} \\
& \leqslant\left\|x_{1}+t\left(x_{2}-x_{1}\right)\right\|^{2}
\end{aligned}
$$

Thus,

$$
t\left\|x_{2}\right\|+(1-t)\left\|x_{1}\right\|=\left\|x_{1}+t\left(x_{2}-x_{1}\right)\right\| .
$$

Taking $t=1 / 2$ in the above equation, we get

$$
\left\|x_{2}\right\|+\left\|x_{1}\right\|=\left\|x_{1}+x_{2}\right\| .
$$

From (2.11), we know that if $x_{1}=0$, then $x_{2}=0$. Hence $x_{1} \neq 0$ and $x_{2} \neq 0$. Since $B$ is strictly convex, according to Lemma 1.1, there exists some $\alpha>0$ such that $x_{1}=\alpha x_{2}$, which contradicts $\left(f_{4}\right)$. This completes the proof.

From $\left(f_{3}\right)$, it is easy to prove the following result.

THEOREM 2.3. Let $A$ be an arbitrary operator acting from the reflexive and smooth Banach space $B$ to $B^{*}$, and $\xi \in B^{*}$. Then the point $x^{*} \in K \subset B$ is a solution of the variational inequality

$$
\langle A x-\xi, y-x\rangle+f(y)-f(x) \geqslant 0, \quad \forall y \in K
$$

if and only if $x^{*}$ is a solution of the following inclusion

$$
x \in \pi_{K}^{f}\left(J(x)-\frac{1}{2}(A x-\xi)\right) .
$$

\section{Applications}

As an application of our results, in this section, we shall study the existence of solutions to the following variational inequality problem: Find $x^{*} \in K$ such that

$$
\left\langle A x^{*}, y-x\right\rangle+f(y)-f\left(x^{*}\right) \geqslant 0, \quad \forall y \in K,
$$

where $K$ is a nonempty, closed and convex subset of the Banach space $B$, and $A: K \rightarrow B^{*}$ and $f: K \rightarrow R \cup\{+\infty\}$ are two mappings. 
DEFINITION 3.1: (KKM mapping) Let $K$ be a nonempty subset of a linear space $X$. A set-valued mapping $G: K \rightarrow 2^{X}$ is said to be a KKM mapping if for any finite subset $\left\{y_{1}, y_{2}, \cdots, y_{n}\right\}$ of $K$, we have

$$
\operatorname{co}\left\{y_{1}, y_{2}, \cdots, y_{n}\right\} \subseteq \bigcup_{i=1}^{n} G\left(y_{i}\right)
$$

where $c o\left\{y_{1}, y_{2}, \cdots, y_{n}\right\}$ denotes the convex hull of $\left\{y_{1}, y_{2}, \cdots, y_{n}\right\}$.

LEMMA 3.1. (FanKKM Theorem [20].) Let $K$ be a nonempty convex subset of a Hausdorff topological vector space $X$ and let $G: K \rightarrow 2^{X}$ be a KKM mapping with closed values. If there exists a point $y_{0} \in K$ such that $G\left(y_{0}\right)$ is a compact subset of $K$, then $\bigcap_{y \in K} G(y) \neq \emptyset$.

THEOREM 3.1. Let $K$ be a nonempty, closed and convex subset of a reflexive and smooth Banach space $B$ with dual space $B^{*}$. Let $A: K \rightarrow B^{*}$ be a continuous mapping and $f: K \rightarrow R \cup\{+\infty\}$ be proper, convex, lower semi-continuous, and bounded from below. Let there exist an element $y_{0} \in K$ such that

$$
\left\{x \in K: 2\left\langle J x-\frac{1}{2} A x, y_{0}-x\right\rangle+\|x\|^{2}+f(x) \leqslant\left\|y_{0}\right\|+f\left(y_{0}\right)\right\}
$$

is a compact subset of $K$. Then the variational inequality (3.1) has a solution.

Proof: From Theorem 2.3, we only need to prove that the following inclusion has a solution,

$$
x \in \pi_{K}^{f}\left(J(x)-\frac{1}{2} A x\right) .
$$

Define a set-valued mapping $W: K \rightarrow 2^{K}$ as follows:

$$
W(y)=\left\{x \in K: G\left(J x-\frac{1}{2} A x, x\right) \leqslant G\left(J x-\frac{1}{2} A x, y\right)\right\} .
$$

Clearly, for each given $y \in K, W(y)$ is nonempty. Next we prove that $W(y)$ is closed for each given $y \in K$. Suppose $\left\{x_{n}\right\} \in W(y)$ and $x_{n} \rightarrow x_{0}$ as $n \rightarrow \infty$. Then,

$$
G\left(J x_{n}-\frac{1}{2} A x_{n}, x_{n}\right) \leqslant G\left(J x_{n}-\frac{1}{2} A x_{n}, y\right)
$$

and so

$$
-2\left\langle J x_{n}-\frac{1}{2} A x_{n}, x_{n}\right\rangle+\left\|x_{n}\right\|^{2}+f\left(x_{n}\right) \leqslant-2\left\langle J x_{n}-\frac{1}{2} A x_{n}, y\right\rangle+\|y\|^{2}+f(y) .
$$

Since $J$ and $A$ are continuous and $f$ is lower-semi-continuous,

$$
-2\left\langle J x_{0}-\frac{1}{2} A x_{0}, x_{0}\right\rangle+\left\|x_{0}\right\|^{2}+f\left(x_{0}\right) \leqslant-2\left\langle J x_{0}-\frac{1}{2} A x_{0}, y\right\rangle+\|y\|^{2}+f(y) .
$$


Hence,

$$
G\left(J x_{0}-\frac{1}{2} A x_{0}, x_{0}\right) \leqslant G\left(J x_{0}-\frac{1}{2} A x_{0}, y\right)
$$

which implies that $x_{0} \in W(y)$.

Next we prove that the map $W: K \rightarrow 2^{K}$ is a KKM mapping in $K$. In fact, suppose $y_{1}, y_{2}, \cdots, y_{n} \in K$ and $0<\lambda_{1}, \lambda_{2}, \cdots \lambda_{n} \leqslant 1$ with $\sum_{i=1}^{n} \lambda_{i}=1$. Let $v=\sum_{i=1}^{n} \lambda_{i} y_{i}$. By property (viii) of $G$, we have

$$
\begin{aligned}
G\left(J v-\frac{1}{2} A v, v\right) & =G\left(J v-\frac{1}{2} A v, \sum_{i=1}^{n} \lambda_{i} y_{i}\right) \\
& \leqslant \sum_{i=1}^{n} \lambda_{i} G\left(J v-\frac{1}{2} A v, y_{i}\right)
\end{aligned}
$$

This implies that

$$
G\left(J v-\frac{1}{2} A v, v\right) \leqslant \max _{1 \leqslant i \leqslant n} G\left(J v-\frac{1}{2} A v, y_{i}\right)
$$

Hence there exists at least one number $j=1,2, \cdots, n$, such that

$$
G\left(J v-\frac{1}{2} A v, v\right) \leqslant G\left(J v-\frac{1}{2} A v, y_{j}\right)
$$

that is, $v \in W\left(y_{j}\right)$. Thus, $W$ is a KKM mapping.

If $x \in W\left(y_{0}\right)$, then $G(J x-(1 / 2) A x, x) \leqslant G\left(J x-(1 / 2) A x, y_{0}\right)$. From the definition of $G$, we have

$$
\begin{aligned}
\left\|J x-\frac{1}{2} A x\right\|^{2}-2\left\langle J x-\frac{1}{2} A x,\right. & x\rangle+\|x\|^{2}+f(x) \\
& \leqslant\left\|J x-\frac{1}{2} A x\right\|^{2}-2\left\langle J x-\frac{1}{2} A x, y_{0}\right\rangle+\left\|y_{0}\right\|^{2}+f\left(y_{0}\right) .
\end{aligned}
$$

Simplifying the above inequality, we have

$$
2\left\langle J x-\frac{1}{2} A x, y_{0}-x\right\rangle+\|x\|^{2}+f(x) \leqslant\left\|y_{0}\right\|^{2}+f\left(y_{0}\right) .
$$

We get that

$$
W\left(y_{0}\right)=\left\{x \in K: 2\left\langle J x-\frac{1}{2} A x, y_{0}-x\right\rangle+\|x\|^{2}+f(x) \leqslant\left\|y_{0}\right\|+f\left(x_{0}\right)\right\} .
$$

By condition (3.2), we know that $W\left(y_{0}\right)$ is compact. It follows from Lemma 3.1 that $\bigcap_{y \in K} W(y) \neq \emptyset$ and so there exits at least one $x^{*} \in \bigcap_{y \in K} W(y)$, that is,

$$
G\left(J x^{*}-\frac{1}{2} A x^{*}, x^{*}\right) \leqslant G\left(J x^{*}-\frac{1}{2} A x^{*}, y\right), \quad \forall y \in K
$$


From the definition of the generalised $f$-projection operator $\pi_{K}^{f}$, we have

$$
x^{*} \in \pi_{K}^{f}\left(J x^{*}-\frac{1}{2} A x^{*}\right)
$$

This completes the proof.

TheOREM 3.2. Let $B$ be a reflexive and smooth Banach space with dual space $B^{*}$ and $K$ be a nonempty, closed and convex subset that contains the origin $\theta$ of $B$. Let $A: K \rightarrow B^{*}$ be a continuous mapping and $f: K \rightarrow R \cup\{+\infty\}$ be proper, convex, lower semi-continuous, and bounded from below. If the set

$$
\left\{x \in K:\langle A x, x\rangle+f(x) \leqslant\|x\|^{2}+f(\theta)\right\}
$$

is compact, then variational inequality (3.1) has a solution.

Proof: Taking $y_{0}=\theta$ in condition (3.2) and noticing that $\langle J x, x\rangle=\|x\|^{2}$, it follows from condition (3.3) that all conditions of Theorem 3.1 hold. Thus Theorem 3.1 implies that the conclusion of Theorem 3.2 hold. This completes the proof.

From Theorem 3.2, it is easy to have the following result.

Theorem 3.3. Let $B$ be a reflexive and smooth Banach space with dual space $B^{*}$ and $K$ be a nonempty, closed and convex cone of $B$. Let $A: K \rightarrow B^{*}$ be a continuous mapping and $f: K \rightarrow R \cup\{+\infty\}$ be proper, convex, lower semi-continuous and bounded from below. If

$$
\left\{x \in K:\langle A x, x\rangle+f(x) \leqslant\|x\|^{2}+f(\theta)\right\}
$$

is compact, then variational inequality (3.1) has a solution.

\section{REFERENCES}

[1] Y. Alber, 'Generalized projection operators in Banach spaces: properties and applications', Funct. Differential Equations Israel 1 (1993), 1-21.

[2] Y. Alber, Metric and generalized projection operators in Banach spaces: properties and applications, Lecture Notes in Pure and Appl. Math. 178 (Dekker, New York, 1996).

[3] Y. Alber, A. Iusem and M. Solodov, 'Minimization of nonsmooth convex functionals in Banach spaces', J. Convex Anal. 4 (1997), 235-254.

[4] Y. Alber, R. Burachik and A. Iusem, 'A proximal point method for nonsmooth convex optimization problems in Banach spaces', Abstr. Appl. Anal. 2 (1997), 97-120.

[5] Y. Alber and S. Guerre-Delabriere, 'On the projection methods for fixed point problem', Analysis 21 (2001), 7-30.

[6] Y. Alber and A. Iusem, 'Extension of subgradient techniques for nonsmooth optimization in Banach spaces', Set-Valued Anal. 9 (2001), 315-335.

[7] Y. Alber, 'Proximal projection method for variational inequalities and Cesáro averaged approximations', Comput. Math. Appl. 43 (2002), 1107-1124. 
[8] Y. Alber, 'On average convergence of the iterative projection methods', Taiwanese J. Math. 6 (2002), 323-341.

[9] Y. Alber and M. Nashed, 'Iterative-projection regularization of unstable variational inequalities', Analysis 24 (2004), 19-39.

[10] C. Baiocchi and A. Capelo, Variational and quasi-variational inequalities, application to free boundary probles (Wiley, New York/London, 1984).

[11] K. Fan, 'A generalization of Tychonoff's fixed point theorem', Math. Ann. 142 (1961), 305-310.

[12] F. Giannessi and A. Maugeri, Variational inequalities and network equilibrium problems (Plenum, New York, 1995).

[13] G. Isac, Complementarity problems, Lecture Notes in Math. 1528 (Springer-Verlag, Berlin, 1992).

[14] G. Isac, V.M. Sehgal and S.P. Singh, 'An alternate version of a variational inequality', Indian J. Math. 41 (1999), 25-31.

[15] W.K. Kirk and B. Sims, Handbook of metric fixed point theory (Kluwer Academic Publishers, Dordrecht, 2001).

[16] J. Li, 'On the existence of solutions of variational inequalities in Banach spaces', J. Math. Anal. Appl. 295 (2004), 115-126.

[17] J. Li, 'The generalized projection operator on reflexive Banach spaces and its applications', J. Math. Anal. Appl. 306 (2005), 55-71.

[18] W. Takahashi, Nonlinear functional analysis (Yokohama Publishers, Yokohama, 2000).

[19] M.M. Vainberg, Variational methods and method of monotone operators (John Wiley \& Sons, New York, 1973).

[20] G.X.Z. Yuan, KKM theory and applications in nonlinear analysis (Marcel-Dekker, New York, 1999).

Department of Mathematics

Sichuan University

Chengdu, Sichuan, 610064

Peoples Republic of China

e-mail: nanjinghuang@hotmail.com 